Ralf Simon

\title{
Raabes literarische Historik
}

I.

Historik ist nach Johann Gustav Droysen die methodengeleitete Grundlagenreflexion des akademischen Faches Geschichte. Von Raabes Historik sprechen zu wollen, heißt also, dem Dichter eine reflektierte Methodologie der historischen Erkenntnis zu unterstellen. Man kann zweifelsohne von Raabes Geschichtsbild sprechen, von seiner Poetik der Erinnerung oder von historistischen Denkweisen, aber Raabe eine Historik zuzuschreiben, trägt seinen literarischen Formen der historischen Vergegenwärtigung die Idee eines Kanons von reflexiv begründeten Methoden zu. Raabe hat eine solche Historik nicht geschrieben, und also kann man mit allem Recht den Begriff einer Raabeschen Historik abweisen. Andererseits gehört es zur Struktur auch der erzählenden Prosadichtung, dass sie grundsätzlich in einer Sprache zweiter Ordnung verfasst ist. Sie stellt die Sprache, in der sie ihre artifiziellen Vergegenwärtigungen vollzieht, immer auch als solche heraus, sie markiert ihr Procedere, sie ist, indem sie im Vorgang des Erzählens aufzugehen scheint, auch zugleich die Reflexion dieses Erzählvorganges.

$\mathrm{Zu}$ Raabes erzählerischer Welt behaupte ich diese drei Eigentümlichkeiten: Raabe erzählt historisch in einem qualifizierten Sinne, als Zeitgenosse des Historismus; er ist unter den sogenannten Realisten zusammen mit Stifter derjenige, dessen Erzählungen die sprachliche Selbstexposition am deutlichsten markieren. Deshalb ist drittens seine historische Thematik immer auch die Reflexion des Historischen selbst: ,erzählte Geschichte‘ ist immer auch erzähltes Erzählen im Sinne von narrativ reflektierter Narration. Derart ist von Raabes Historik zu sprechen, und zwar um so mehr, als in seinem Werk eine in der literarischen Form vorgetragene Systematik der erzählerischen Vergegenwärtigungen des Historischen nachzuweisen ist. Die immanente poetologische Reflexion des Erzählvorganges bei Raabe lässt sich also - so die These - als Poetik des Historischen lesen, als in praxi vollzogene Metahistory. Ich möchte sie im Folgenden literarische Historik nennen.

II.

Droysens Historik ${ }^{1}$ gliedert die Methodenlehre in Heuristik, (Quellen-)Kritik und Interpretation. Der Begriff der Interpretation wird dabei vierfach ausdifferenziert:

1 Johann Gustav Droysen: Historik. Vorlesungen über Enzyklopädie und Methodologie der Geschichte. Hg. von Rudolf Hübner, München, 6. Aufl. 1971. 
in pragmatische Interpretation, Interpretation der Bedingungen, psychologische Interpretation und Interpretation der Ideen. ${ }^{2}$ Aufschlussreich für die Verortung von Raabes literarischer Historik im Rahmen der Unterscheidungen Droysens ist das Verhältnis der psychologischen Interpretation zur Interpretation der Ideen. Die Geschichtswissenschaft kann und will nicht in den eigensten Kreis der jeweiligen historischen Persönlichkeit ${ }^{3}$ eindringen, so Droysen. Vielmehr betrachtet sie ihr Tun als Moment in der Bewegung der sittlichen Mächte. ${ }^{4}$ Diese darzustellen, also den Gehalt der geschichtlichen Ereignisse einer Zeit als „Komplex von Verwirklichungen aller sittlichen Mächte ${ }^{* 5} \mathrm{zu}$ verstehen, ist das eigentliche Ziel der so gestaffelten Interpretationstätigkeit des Historikers. ${ }^{6}$ Droysen markiert den Übergang der psychologischen Interpretation zu der der Ideen als Lücke: „Die Interpretation der Ideen tritt in die Lücke, welche die psychologische läßt.“7 Innerhalb einer Methodologie der Geschichtswissenschaft, welche ihr Gebiet als „Kosmos der sittlichen Welt" ${ }^{\text {"K }}$ bestimmt und die sittlichen Mächte als Kollektivformen denkt (Familie, Volk, Sprache, Künste, Religionen, Recht etc.), führt in der Tat kein wissenschaftlicher Weg von den psychologischen Bedingungen des individuellen Handelns zur Erkenntnis des Geschichtsverlaufs. Aber eine literarische Historik findet gerade hier ihren Ausgang. In der literarischen Erzählung wird die thematische Historie aus dem Konstruktionspunkt der psychologischen Exposition heraus entwickelt. Der Erzähler sieht das Handeln mit den Augen seiner Figuren, von innen nach außen - eine Blickweise, die der des Historikers auf die grundsätzlichste Weise widerspricht. Gleichwohl gilt aber auch für die literarisch erzählte Welt, dass sie als historische Erzählung an den Gang der Ereignisse gebunden bleibt. Diesem Gang als solchem kann die Literatur keine psychologische Interpretation angedeihen lassen. Sie kann aber den Gang der Geschichte insofern neu erfinden, als sie über die Perspektive der fiktionalen Figurenpsychologie verfügt, zumal, wenn Raabe sein Personal auf der Ebene derer ansiedelt, die nicht zu den Mächtigen gehören, von denen manche Diskurse behaupten, sie würden die Geschichte machen.

Auch für Raabe entsteht das Problem der Lücke zwischen der Psychologie des Individuellen und der Allgemeinheit der geschichtlichen Kollektivmächte. Es bedrängt ihn radikaler als Droysen, denn während dieser nicht einmal die Möglichkeit erwägt, je an diese Wurzel des Individuums heran reichen zu können, ist Raabes literarische Narration notwendig aus dem Konstruktionspunkt des Individuellen heraus entwickelt. Als Folge des derart verschärften Konflikts

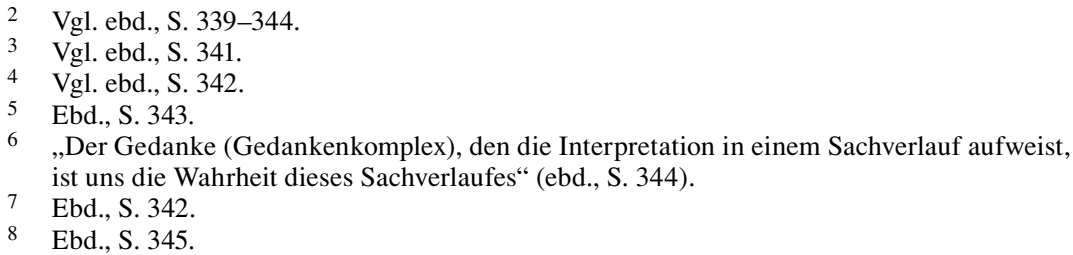


zwischen Psychologie und Kollektivform entsteht für Raabes Geschichtsnarration das Problem der Gerechtigkeit. ${ }^{9}$

Raabes Texte handeln von Individuen, deren Verhalten inmitten einer katastrophisch verlaufenden Geschichte aus dem individuellen, oft schon auf den idyllischen Nahbereich reduzierten und enttäuschten Verlangen nach Glück motiviert ist. Diesem Glücksverlangen steht das Unheil der Geschichte als vernunftloser Ereignisfolge von Zerstörungsakten gegenüber. Die literarische Erzählung sucht zwischen beiden Einheiten - der des Individuums und der des allgemeinen Unheils - eine Vermittlung zu finden. Bei Raabe sind es nicht selten die alten Männer als Figurationen der Gerechtigkeit, die jungen Liebespaaren helfen, sich in den Katastrophen der Kriegswirren zurecht zu finden. Mit Jacob Burckhardts impliziter Historik ${ }^{10}$ kann man diese Vermittlung als Gleichgewicht bezeichnen. Gleichgewicht, bei Burckhardt die verwandelte Nemesis-Kategorie des späten Herder, ${ }^{11}$ ist freilich keine Kategorie, die auf vordergründige, nämlich themati-

9 Anselm Haverkamp ist es zu verdanken, den Begriff der Gerechtigkeit vehement in das Zentrum der literaturwissenschaftlichen Gedächtnisforschung gestellt zu haben. Haverkamp erinnert daran, dass der eigentliche Sinn der zuweilen positivistischen Konjunktur des Gedächtnisthemas im Begriff der Gerechtigkeit liegt. Erinnerungsarbeit und Gedächtniskulturen werden nicht als Selbstzweck betrieben, sondern um der Gerechtigkeit willen. Menschen behalten die Dinge im Gedächtnis und wollen sich ihrer erinnern, um später einen gerechten Ausgleich vollziehen zu können. Die Geschichtswissenschaft wäre, weil sie Erinnerungsarbeit ist, diesem Gedächtnisbegriff einzugliedern. Haverkamp insistiert aber darauf, dass es insbesondere der poetische Text ist, welcher stets auf die im Laufe der Interpretationsgeschichte an ihn ergehenden Fragen eine Antwort zu geben in der Lage ist. So rückt die poetische Textur schon in sich selbst, aufgrund ihrer internen Struktur, infiniten Sinn zu bergen, an die Stelle einer metaethisch begriffenen Gerechtigkeit. Vgl. Anselm Haverkamp: Figura cryptica. Theorie der literarischen Latenz, Frankfurt/Main 2002, S. 149-162.

10 Ich bezeichne die „Weltgeschichtlichen Betrachtungen“ (1905 aus dem Nachlass veröffentlicht) als Jacob Burckhardts Pendant zu Droysens Historik. Zwar handelt es sich hier nicht im engeren Sinne um eine Methodik des Geschichtsstudiums, sondern vielmehr um den Versuch, die Konstituentien von Geschichte, verstanden als theoretischer Gegenstand, zu bedenken. Burckhardts Text trifft sich aber mit Droysens Ausführungen, wo dieser nicht von der Methodik, sondern von der Systematik handelt und die sittlichen Mächte als Konstituentien der Geschichte bespricht.

11 Dass Burckhardt ein intensiver Herder-Leser war, ist bekannt. Herder entwickelt im XV. Buch seiner „Ideen zur Philosophie der Geschichte der Menschheit" eine Theorie des Gleichgewichts (Terminus: „Billigkeit“), die er dann später, in seinem „Nemesis“-Aufsatz und in seinem Zeitschriftenprojekt „Adrastea“ weiterentwickelt. Nemesis ist bei Herder nicht mehr die erbarmungslose Rachegöttin, sondern vielmehr die Göttin des Ausgleichs, der Austarierung. Sie übernimmt die geschichtliche Verlust- und Gewinnbilanzierung, um für den schlussendlichen Ausgleich besorgt zu sein, welcher die Geschichte in die narrative Homöostase lenkt. Vgl. zu diesen Begriffen bei Herder Ralf Simon: Apokalyptische Hermeneutik. Johann Gottfried Herder: Maran Atha, Geschichtsphilosophie, Adrastea. In: Herder Jahrbuch/ Herder Yearbook 1998, S. 27-52, bes. S. 42-45, und Ralf Simon: Historismus und Metaerzählung. Methodologische Überlegungen zur Erzählbarkeit von Geschichte in Herders Geschichtsphilosophie. In: Nationen und Kulturen. Zum 250. Geburtstag Johann Gottfried Herders. Hg. von Regine Otto, Würzburg 1996, S. 77-95, bes. S. 88-94. 
sche Art und Weise dem individuellen Begehren gegen die destruktiven Kräfte zur Durchsetzung verhelfen würde. Es handelt sich vielmehr um eine metaethische Kategorie, die einerseits narratologisch auf eine Tiefenebene abzielt, die man als die strukturale Homöostase des Erzählprozesses bezeichnen kann. Andererseits, als Kategorie der Historik, meint Gleichgewicht oder Nemesis die Einschätzung der - um mit Burckhardt zu sprechen - ,großen weltgeschichtlichen Ökonomie“, ${ }^{12}$ deren vermuteter Endzweck darin besteht, „,bloße Macht in Ordnung und Gesetzlichkeit umzuwandeln“.13

Diese Umwandlung, so lautet die These, ist struktural immer schon am Werke, wenn überhaupt der Prozess der Narration im Gange ist. Egal, welche Katastrophe thematisch verhandelt wird, egal auch, ob eine Erzählung ein gutes oder ein schlechtes Ende hat: Immer und notwendig folgt das Erzählen einer Logik der Homöostase, des Ausgleichs, des Tausches der Narrateme. Die Begründung für dieses Theorem ist einfach und elementar. Das Erzählen ist ein dreistufiger Prozess, in dem auf eine Ausgangslage, die in den formalisierenden Narratologien meist als semantische Opposition dargestellt wird, ${ }^{14}$ eine Vermittlung und schließlich eine Lösung folgt. ${ }^{15}$ Die Lösung muss sich formaliter - also nicht inhaltlich als glückliches Ende - als Antwort auf die Ausgangslage darstellen. Wir sprechen, auch in der Alltagssemantik, dann von einer Erzählung, wenn wir eine derart zusammenhängende Sequenz mitteilen. Würde eine solche Lösung fehlen, so läge vielleicht eine Aufzählung vor, aber keine Narration.

Das in gepflegten Erzählungen grundsätzliche Eingebundensein der Narrateme in eine causa finalis zieht einige narratologische Schlussfolgerungen nach sich. Indem eine Ausgangslage in eine Vermittlung gezogen wird, also etwa oppositionelle Terme auf die Zeitachse gelegt und durch Zwischenterme aus der direkten semantischen Opposition befreit werden, findet zugleich eine Transformation statt. Eine Erzählung hat immer ein Thema, über welches es ,etwas ‘ zu erzählen gibt, und die Erzählung besteht darin, dieses Etwas als Veränderung des Thematischen so auszuführen, dass die Veränderung wiederum in das Thematische eingeschrieben wird. ,Veränderung ' in diesem allgemeinen Sinne heisst vor allem, dass es einen Weltkontakt gibt, ein Ereignis, ${ }^{16}$ das eine andere und neue Dimension einbringt. Einem Menschen in einer bestimmten Lage passiert ,etwas'; dieses Etwas macht die Vermittlung zu der anfänglichen stabilen Lage notwendig; es folgt eine Reihe von kleineren Handlungen, solange bis die Person

12 Jacob Burckhardt: Weltgeschichtliche Betrachtungen. In: Ders.: Das Geschichtswerk, Frankfurt/Main 2007, Bd. 1, S. 957.

13 Ebd., S. 958.

14 Algirdas Julien Greimas: Strukturale Semantik. Methodologische Untersuchungen, Braunschweig 1971, bes. Kap. 2 („Die elementare Struktur der Bedeutung“).

15 Claude Bremond: Die Erzählnachricht. In: Literaturwissenschaft und Linguistik III. Hg. von Jens Ihwe, Frankfurt/Main 1972, S. 177-217.

16 Jurij Lotman: Die Struktur literarischer Texte, München, 2. Aufl. 1981, bes. Kapitel VIII. 
eine neue Lage gefunden hat, in der dieses Etwas einen Platz hat. ${ }^{17}$ Die Reihe der kleineren Handlungen - also die Vermittlung zwischen Ausgangslage und Erzählende - ist aber ein Geschehen des Austausches und damit des Abgleichens, Vergleichens und des Herstellens eines Gleichgewichts. Die Person agiert, indem sie ihre Lage und ihre Interessen mit den Bedingungen der Welt abgleicht. Sie übereignet der Welt einen Teil der eignen Aktivität, als Reaktion auf die Aktivität, die aus der Welt auf sie zugekommen ist. Indem derart auf ein Ereignis eine Reaktion folgt, stellt sich jedes Mal die Frage, ob die Reaktion dem Ereignis gerecht wird, ob sie dem Entgegenkommenden mit einer gleichen Macht antwortet, ob sie also den gestellten Ansprüchen standhalten kann. Die Person misst sich an der Welt durch die Handlung. Die erzählten Handlungseinheiten, die Narrateme, sind also gewissermaßen Tauscheinheiten: Auf ein Ereignis, das ihm zustößt, zahlt der Handelnde in der Währung seines Reichtums, nämlich seiner Handlungsmöglichkeiten. Die in dem Wort Erzählen steckende Vorstellung von der Zahl und dem Zählen meint genau dies: das quantifizierende Abtragen einer anfänglichen qualitativen Initiative mit dem Ziel, vermöge der narrativen Akkumulation zu einer qualitativen Antwort zu kommen. In diesem ganz elementaren Sinne ist jedes Erzählen ein Prozess des Gleichgewichtfindens, des Austauschs mit der Welt so lange, bis die individuellen Intentionen sich ins Einvernehmen mit den äußeren Bedingungen gesetzt haben.

Erzählen, so lautet das Fazit, ist ein permanentes Ausgleichen und Abwägen. Jede Handlungseinheit wird gewissermaßen daraufhin geprüft, ob sie tauglich ist, auf die Störung der Ausgangslage eine Antwort oder ein Teil einer Antwort zu sein. ${ }^{18}$ In diesem grundsätzlichen narratologischen Sinne ist die Erzählung permanent mit der Herstellung von Gleichgewicht, Homöostase, Nemesis beschäftigt. Die regulative Idee des Gleichgewichtes als metaethischer Kategorie der Historik ist insofern auch immer ein Implikat der Erzählung.

Man sieht hier deutlich, dass die Form der Narrativität in einer Spannung zur Offenheit der Geschichte steht. Droysens Begriff der Lücke zwischen psychologischer Interpretation und Interpretation der Ideen (s. o.) hat diese Widersprüchlichkeit ebenso artikuliert, wie Jacob Burckhardt, dessen letztes Kapitel aus den Weltgeschichtlichen Betrachtungen über „Glück und Unglück in der

17 Auch diese topologisierende Formulierung ist ganz formal gemeint: Der ,Platz', der durch die Erzählung dem Ereignis, dem vorgefallenen Etwas bereitet wird, muss nicht in einer Integration bestehen. Auch eine scheiternde Integration kann den narrativen Platz bestimmen: als Erzählung, die ihr Fazit auf der inhaltlichen Ebene darin findet, ein Sujetereignis nicht mit der Ausgangslage vermitteln zu können.

18 Wenn Jacob Burckhardt in seinen „Weltgeschichtlichen Betrachtungen“ auf die Frage nach dem Glück in der Weltgeschichte die Antworten vor allem als ein „Urteil aus Ungeduld“ (Burckhardt [wie Anm. 12], S. 951) bezeichnet, als eine zu schnelle Urteilsbildung, die sich scheut, den mühsamen Weg der wissenschaftlichen Anstrengung zu gehen, so mag er damit Recht haben. Aber er reflektiert nicht die narrative Energie, die jeder begonnenen erzählerischen Artikulation eine immanente causa finalis einschreiben muss, sofern sie überhaupt Erzählung sein will. 
Weltgeschichte "19 grundsätzlich daran krankt, dass es eine narratologische Frage stellt, aber den Vollzug der Narration aus Gründen der wissenschaftlichen Solidität verweigert. Es entsteht ein Skeptizismus, der sich als Stilgeste des gediegenen Rückzugs darstellt. Immer wieder spielt Burckhardt dieselbe Frage nach der Narrativität der Geschichte als unausweichliche Frage ein, um die Antwort als vorwissenschaftliche Bedürfnisbefriedung abzuweisen. - Die intelligentere Antwort auf diesen Skeptizismus, der sich nur oberflächig durch seinen Stil salvieren kann, ist in einer reflexiven Poetik der Narrativität des Historischen zu finden. Aus diesem Kontext heraus ist Raabe zu lesen: als poetologische Metahistorik der Historik.

III.

Die nach wie vor elementare wie hochkomplexe Frage lautet: Wie ist Geschichte auf dem Niveau der narratologischen Reflexion zu erzählen? Ich möchte im Folgenden eine reflexionslogische Systematik der Geschichtsnarration vorschlagen. $\mathrm{Zu}$ unterscheiden sind drei Ebenen: das Erzählen als einfache Narration der Ereignisse, das Erzählen ausgehend von der Logik gewusster und explizit gemachter Programme, das Erzählen als Kontingenzreflexion des Erzählprozesses.

Bevor dieser Gedanke ausgeführt wird, soll die hier vorgeschlagene Systematik näher begründet werden. Sie orientiert sich an der im Neukantianismus entwickelten Logik der geltungstheoretischen Selbstreflexion, welche immer nur dreistufig sein kann: ${ }^{20}$

III.1. Reflexion über die Welt ist zunächst und erstens unmittelbare Sachaussage, als Bestimmung von Objektivität. Über die Dinge werden Eigenschaften ausgesagt und in der Abgleichung mit anderen Aussagen über andere Eigenschaften begründet.

III.2. Reflektiert man die Tätigkeit der Objektbestimmung geltungstheoretisch, so befindet man sich auf der Ebene einer zweiten Reflexion in Relation zur ersten Reflexion qua Sachaussage. Man wird die Strukturen dieser Objektbestimmungen zu analysieren haben, also sich diejenigen Kategorien zum Gegenstand machen, welche objektformierend wirken.

19 Burckhardt (wie Anm. 12), S. 947-963.

20 Grundsätzlich ist hier zu betonen, dass ein systematisches und kein historisches Argument entwickelt wird. Natürlich ließe sich über die zeitliche Nähe des Neukantianismus (spätes 19. Jahrhundert) zu Raabe spekulieren, aber die Einflüsse werden spärlich sein und keinesfalls zu der hier entwickelten Systematik führen. Die grundsätzliche Struktur einer literarischen Reflexion der Geschichtsnarration ist deshalb auch als Beitrag zu einer allgemeinen Narratologie zu lesen. Freilich, die These lautet, dass Raabes Geschichtserzählungen in einer spezifischen und ausgezeichneten Weise zu dieser allgemeinen Narratologie stehen. 
III.3. Wird nun auch diese Ebene einer weiteren Geltungsreflexion - nunmehr in dritter Potenz - unterzogen, dann kann es nicht mehr um Objektbestimmung (erste Ebene) und nicht mehr um die kategoriale Logik von Objektbestimmungen (zweite Ebene) gehen, sondern nur noch um die Selbstreferenzen des Denkens als solchem. Diese dritte Reflexion kann nicht mehr durch eine weitere Potenz reflektiert werden, da es gegenüber der allgemeinen Gesetzlichkeit des Denkens keine einsetzende Metainstanz im Denken mehr geben kann. Die Philosophie würde hier die Logik, verstanden nach Hegel, ansetzen, denn die fundierende Begründung der objektbestimmenden Kategorien ist in derjenigen Sphäre zu suchen, in der die reinen Prinzipien für alle möglichen Objektbestimmungen überhaupt zu finden sind. ${ }^{21}$

Versucht man eine Systematik der narrativen Selbstreflexion aus der skizzierten Dreistufigkeit der Reflexion als solcher abzuleiten, so kann es offenkundig nur drei wesentliche Schritte geben:

Ad III.1. Die elementare Erzählung besteht in der singulären Verbindung von Narratem zu Narratem. Sie ist die ganz auf die Seite des Vorhandenen geworfene narrative Verbindung der Dinge durch die Erzählung ihrer metonymischen Relationalitäten und besteht aus singulären Narratemverbindungen (,und dann folgte auf a das Ereignis b').

Ad III.2. Wenn sich das Erzählen aus der puren Praxis des bloßen, jeweils ad hoc motivierten Verknüpfens löst und sich seiner als eigene Form bewusst wird, entsteht die zweite Erzählebene als Kunst der Erzählung. Sie vollzieht die Konvergenz von Motivation-von-vorne und Motivation-von-hinten (, auf a folgt b, weil der Weg zum Ziel über die Reihe $\mathrm{a}-\mathrm{b}$ - etc. geht'). Erzählen wird hier zum Argument: Die Rhetorik zählt ja, so sei erinnert, die narratio zum argumentum. Argumentativ ist das Erzählen, wenn es ein Ziel herleiten will, aber eine Kunst wird es, wenn dieses Ziel nicht deshalb erreicht wird, weil es in einer petitio principii supponiert wurde, sondern weil es gleichsam naturwüchsig aus der eigenen Tendenz der Dinge zu erwachsen scheint. Erzählen als argumentum folgt also Erzählprogrammen, z. B. der Idee, Geschichte folge einem göttlichen Vorsehungsplan oder resultiere aus dem Gewaltzusammenhang der kollektiven Mächte. Formalistischer betrachtet, könnten die objektformierenden Kategorien der Narrativität rhetorisch als Metapher, Metonymie, Synekdoche oder Ironie erscheinen, bezüglich des artikulierenden Registers als Romanze, Komödie, Tragödie oder Satire und bezüglich des Ideologems als Anarchismus, Konservatismus, Radikalismus oder Liberalismus. Nach diesem

21 Vgl. zur Auffaltung dieser Systematik Hans Wagner: Philosophie und Reflexion, München und Basel, 3. Aufl. 1980. 
Kategorienset bestimmt Hayden White ${ }^{22}$ die Erzählformen des Historismus. Es handelt sich offenkundig um eine narratologische Analyse auf der zweiten Ebene der geltungstheoretischen Reflexion der Geschichtserzählung.

Ad III.3. Die dritte Erzählebene kann als Reflexion der zweiten nur die narrativ vollzogene Analyse dieser kunstvollen argumentatio sein. Denn das Universum der Erzählung bleibt an die Form der Narrativität gebunden. Die Erzählung kann nicht ummittelbar die reine Prinzipiensphäre betreten. Die dritte Ebene der Selbstreflexion kann narratologisch nur relevant sein, wenn es eine Sphäre von metanarrativen Narratologiekategorien gibt. Sie wurden genannt: Gerechtigkeit, Ausgleich, Nemesis. Darstellbar zuhanden des Dispositivs der Narration ist diese Sphäre in der Form der immanenten poetologischen Reflexion, als gegenüber der narrativen Artikulation kontingenzerzeugende Geste der Ironie oder des Humors, als Vollzug narrativ ausgleichender Homöostase. Eine narrativ vollzogene Reflexion der Kunst der Narration ist also nur als narrative Poetologie des Narrativen denkbar, als immanente, sich in sich selbst hinein wendende Reflexion des Erzählens selbst, als Erzählen, das unmittelbar zugleich auch Allegorie des Narrativen ist.

Raabes spätere Texte befinden sich grundsätzlich auf dem Niveau dieser poetologischen Selbstreflexion, sie sind als literarische Historik auf der skizzierten dritten Ebene anzusiedeln.

Zweifelsohne gibt es bei Raabe die Idee einer einfachen Narration der Ereignisse (vgl. III.1.), welche die chronologische Reihenfolge wiederzugeben meint. ${ }^{23}$ Die Chronik - titelgebend für Raabes erstes Buch Die Chronik der Sperlingsgasse - bleibt noch unter dem Niveau der Erzählung, sofern sie die Form einer Liste hat. Auch die Akten - titelgebend für eines der letzten Bücher Raabes: Die Akten des Vogelsangs - bilden als solche keine Erzählung, sondern das, was nach Droysen Quellenmaterial ist und einer Kritik bedarf. Die einfache Narration der Ereignisse braucht schon Formmomente, um überhaupt, gegenüber Chronik und Aktenlage, den Schritt von der Aufzählung zur Erzählung zu machen.

Als einfache Narration kann Geschichte in Analogie zur einfachen Erzählform, wie sie Clemens Lugowski anhand der frühneuzeitlichen Prosa von Wickram rekonstruiert, ${ }^{24}$ dargestellt werden. Ein permanentes Motivieren-von-vorne aus Ad-hoc-Kausalitäten heraus lässt jeweils etwas passieren, weil vorher eine metonymische Nachbarschaft den singulären Anstoß nur für dieses eine Ereignis

22 Hayden White: Metahistory. Die historische Einbildungskraft im 19. Jahrhundert in Europa, Frankfurt/Main 1991, bes. Vorwort und Einleitung, S. 9-62.

23 Um es eigens zu betonen: Die Behauptung ist nicht, dass Raabe elementare Erzählungen zu seinem Euvre zählt. Aber er verhandelt die Idee solcher Erzählformen und benennt sie in seinen Texten.

24 Clemens Lugowski: Die Form der Individualität im Roman, Frankfurt/Main 1976. 
gegeben hat. Man kann eine Reihe solcher jeweils vereinzelter Ad-hoc-Kausalitäten anhand einer Person erzählen, der dieses Geschehen zufällt. Es wäre dann eine Erzählung, die nur durch die thematische Konstanz eines Handlungsträgers oder eines Schauplatzes zusammengehalten wird, aber kein narratives Programm hat. Der alte Begriff der Historia hat z. B. als Stadthistorie die pure Kontingenz des Vorgefallenen an einem Ort verzeichnet. Interessanterweise befindet sich die Mehrzahl der bei Raabe nachweisbaren Stellen zum Begriff Historie oder Historia wortsemantisch auf dem Niveau dieser vormodernen Bedeutung von Historie. ${ }^{25}$ Freilich, die erzählten Geschichten folgen bei Raabe nicht mehr dem simplen Erzählfaden bloßer Ad-hoc-Kausalitäten. Aber es gibt dennoch eine Mimesis an genau dieses vormoderne Erzählmodell auf dem einfachsten semiotischen Niveau der erzählerischen Artikulation. In den humoristischen Texten wie Die Gänse von Bützow lässt Raabe programmatisch die lächerlichsten Zufälle eintreten, um eine nicht weniger lächerliche Kampagne angemaßter revolutionärer Heroik ad absurdum zu führen. Die Erzählung gerät so zu einem „Museo aus dem Gang der Dinge“, zur Vorstellung der Welt als „,ein gar jokoses und amüsantes Theatrum“ (BA 9/2, S. 64), ${ }^{26}$ in welchem das Größte vom Kleinsten konterkariert wird. In dieser Erzählung, die natürlich ein Programm besitzt, wird gleichwohl eine permanente durchkreuzende Kontingenz seltsamster Vorfälle inszeniert und damit ein humoristischer Gegentext zum narrativen Gefüge erzeugt. Raabe zitiert mit Begriffen wie Museo, Theatrum und Historia vormoderne Semantiken und evoziert die Vorstellung der kuriosen Exempelsammlung, deren textuelle Exponierung durch ein Erzählprogramm nicht dargestellt werden kann. ${ }^{27}$

Die zweite reflexionslogisch folgende Ebene der Geschichtsnarration kennt Erzählprogramme (vgl. III.2.). Dies impliziert, dass sich das Erzählen als solches seiner selbst bewusst geworden ist. Es stellt sich unter ein artikuliertes Prinzip. Es verfolgt eine Absicht, die causa efficiens wird auf eine causa finalis ausgerichtet, auf ein Motivieren-von-hinten, auf eine Um-zu-Argumentation. Geschichte kann auf diese Weise verschiedensten Erzählprinzipien folgen. Raabe kennt die Narration der geschichtlichen Ereignisse als Exemplifizierung ihrer Sinnlosigkeit, als Exemplifizierung der Kohäsionskraft des Hasses, als Exemplifizierung eines höheren Planes, sei es der Plan der guten Natur oder der einer göttlichen

25 Vgl. zur historischen Semantik von „Geschichte“ und „Historie“: Reinhart Koselleck, Horst Günther, Christian Meier: Geschichte, Historie. In: Geschichtliche Grundbegriffe. Hg. von Otto Brunner, Werner Conze, Reinhart Koselleck, Stuttgart 2004, Bd. 3, S. 593 717.

26 Zitate aus Wilhelm Raabes Werken werden unter Verwendung der Sigle BA (mit Bandnummer und Seitenzahl) nach den jeweils neuesten Bandauflagen der „Braunschweiger Ausgabe“" belegt: Wilhelm Raabe: Sämtliche Werke. 20 Bde. und 5 Ergänzungsbände. Hg. im Auftrag der Braunschweigischen Wissenschaftlichen Gesellschaft von Karl Hoppe und Jost Schillemeit, Göttingen 1960ff.

27 Vgl. auch „Hastenbeck“ (BA 20, S. 146f. u. ö.). 
Ökonomie. ${ }^{28}$ Die in jeder Hinsicht dunkle Erzählung Im Siegeskranze wird als Narration einzig durch die Kraft des Negativen zusammengehalten, und das Odfeld inszeniert eine Kreisfigur als Ausweichmanöver vor einer Schlacht, welche am Ende nur zur inzwischen zerstörten Anfangsidylle zurückkehrt. Raabes Erzählungen geschichtlicher Verläufe oder Ereignisse sind in dieser Weise, schon seit der Chronik der Sperlingsgasse, vor allem Verläufe, die durch das Band der Negativität zusammengehalten werden.

Auf dieser Ebene der Erzählung befindet sich Raabes Prosa auf dem Niveau der für den Historismus üblichen Geschichtsnarration. ${ }^{29}$ Die erzählerische Kunst der großen Geschichtsdarstellungen besteht in der bruchlosen Konvergenz einerseits der ja immer vorhandenen singulären Motivation-von-vorne, andererseits der durch implizite Erzählprogramme gesteuerten Motivation-von-hinten. Wenn Burckhardt die Kultur der Renaissance als ein geschlossenes System mit interner Lebensrhythmik darstellt, so folgt er formaliter einem Programm, welches er freilich, um den deduktiven Zirkelschluss zu vermeiden, immer nur induktiv herleitet. Es handelt sich in der Tat um eine mit hoher Bewusstheit ergriffene Kunst, welche das steuernde Programm immer nur als Resultat des natürlichen Zusammenhangs der Dinge auftreten lassen will. Darin ist formaliter kein Widerspruch zu einer Geschichtsnarration zu sehen, welche bei Raabe (und also mit Schopenhauer) die grundsätzliche Negativität des Geschichtsverlaufs darstellt.

Raabe zitiert mit Begriffen wie Historia, Theatrum, Curiositas ein Beisammensein der Dinge, welches sich als elementare Erzählung beschreiben lässt. Und er zitiert mit dem Verweis auf den göttlichen Heilsplan oder mit dem Schopenhauerschen Pessimismus Modelle einer Narrativität der Geschichte. Er zitiert also mögliche Modelle der narrativen Objektbestimmung, die er wie in Hastenbeck als Idylle erzählt oder wie im Odfeld als zur Metapher sich schließenden Metonymienkreis. ${ }^{30}$ Aber obwohl er expressis verbis die erste und die zweite

28 Der Plan der Vorsehung wird in vielen Texten Raabes zitiert, ich erspare mir eine umfangreiche Belegstellenliste, die sich jederzeit über eine elektronische Recherche erzeugen lässt. Freilich wird das Erzählmodell der providentiellen Steuerung nie affirmativ eingesetzt, sondern immer skeptisch zitiert. Vgl. in diesem Zusammenhang auch die Monographie von Heinrich Detering: Theodizee und Erzählverfahren. Narrative Experimente mit religiösen Modellen im Werk Wilhelm Raabes, Göttingen 1990 (= Palaestra, Bd. 289).

29 Um es genau zu formulieren: Raabes Erzählprogramme sind formaliter ganz im Sinne des Historismus kunstvolle Narrationen, die individuelle Geschicke mit den kollektiven Mächten der Geschichte vermitteln. Aber natürlich unterscheiden sie sich in wesentlichen Eigenschaften vom Historismus. Einerseits sind Raabes Erzählprogramme pessimistischer als die des Historismus, andererseits erlauben es ihm seine literarischen Verfahren, Geschichte von einem anderen Standpunkt aus zu konstruieren. - Diesen Abweichungen wird in meiner Systematik Rechnung getragen, indem Raabe grundsätzlich auf die dritte Ebene der Geschichtsreflexion versetzt wird, also die hier thematische zweite Ebene gleichsam immer nur von der dritten aus formiert wird. Gleichwohl ist die zweite Ebene als solche zu benennen.

30 Jede Metapher ist durch eine Reihe von Metonymien paraphrasierbar. Der große semantische Sprung der Metapher kann auch durch eine lange Reihe von kleinen metonymischen 
Reflexionsstufe der narrativen Artikulation verhandelt, sind seine Erzählungen doch durchgängig auf der hier konstruierten Ebene der dritten, nämlich poetologischen Reflexion des Narrativen überhaupt anzusiedeln (vgl. III.3.). Man kann an diesem Punkt vielleicht behaupten, dass Raabe, verglichen mit Droysen oder Burckhardt, in einem sehr viel qualifizierteren Sinne eine Historik kennt. Denn während die Historiker nur die zweite Reflexionsebene formulieren können und Burckhardt allerhöchstens in der Stilgebärde eine weitere Reflexion markiert, hat Raabe das literarische Privileg, Geschichte in einer potenzierten Reflexionsform, nämlich derjenigen der dekonstruktiven Allegorie der Geschichtserzählung vorzutragen. Er nutzt die ästhetische Lizenz der immanenten Reflexion qua Poetologie, um zielgenau gerade diejenigen Kategorien fiktional zu inszenieren, die innerhalb einer Narratologie der dritten Reflexionspotenz als Theorie unendlich schwieriger zu exponieren sind. Raabe liest nämlich die Historik der zweiten Reflexionsstufe innerhalb einer Poetologie der Kontingenz. Beginnend mit der mittleren Werkphase, aber in die Sichtbarkeit vorangetrieben in seinem Spätwerk, stellen seine Erzählungen immer auch zugleich die Poetik des Erzählens im Modus der Unzuverlässlichkeit der Narration und sogar im Modus ihrer prinzipiellen Falschheit vor.

IV.

Das Odfeld zwingt die Narration in ihren zirkulären Kollaps gerade durch das Kurzschließen von causa efficiens und causa finalis. Die causa finalis des Geschehens besteht in der Motivation der Handelnden, der sich abzeichnenden Schlacht auszuweichen. Das Handeln der Akteure ist also durch eine Zieldefinition gesteuert. Die Realität der erzählten Welt erzwingt aber eine permanente Korrektur der Zielvorgaben, so dass sich eine metonymische Logik der Motivation-von-vorne so ausdauernd vor die Zielrealisierung schiebt, dass am Ende ein Kreislauf stattgefunden hat und die Akteure beim nunmehr zerstörten Ausgangspunkt ankommen. Erzähltheoretisch wird mit diesem Rückfall der causa finalis auf die Kontingenzreihe von Ad-hoc-Ereignissen die Kunst der Erzählung unterwandert. Dementiert wird, dass die causa finalis die unsichtbare Macht hätte, die causa efficiens unbeschadet ihrer induktiven Logik zum Ziel zu bringen. Die historistische Kunst der Erzählung strandet bei der einfachsten narrativen Form. Die bloße metonymische Verknüpfung erzeugt keine Sinnfigur, sondern einen Kreis um eine mögliche Schlacht mit dem Ergebnis, dass der anfänglich intakte Ausgangspunkt zerstört ist. Raabes Erzählung einer Gruppe

Nachbarschaften erzeugt werden. Vgl. dazu Umberto Eco: Semantica della metafora In: Ders.: Le forme del contenuto, Mailand 1971. Raabes „Odfeld“ exerziert genau diese Bewegung durch. Der Kreisgang bildet keine Metapher, sondern das Zurücksinken auf die Metonymie. Man könnte dies, auf der tropologischen Ebene, „Realismus“ nennen. 
von Flüchtenden führt die Erzählung auf den Nullpunkt der narrativen Semiose zurück. Der reale Kreis, der gelaufen wird, ist mitnichten eine Metapher des geschlossenen Werks, ist keine erfüllte Vermittlungsfigur, sondern vielmehr eine poetologische Reflexion jener narrativen Sinnfiguren, die in der historistischen Sinnkonstruktion auf der hier konstruierten zweiten Reflexionsstufe der Narration affirmiert werden. Als poetologische Reflexion dieser zweiten Stufe (also als Tun auf der dritten Reflexionsebene) ist das Odfeld ein radikaler Text: eine Dekonstruktion historischen Sinns mit den Mitteln einer poetologisch-narrativen Historik. Raabe schaut in das Auge des geschichtlichen Sturms und sieht den Fluchtgrund der vermuteten Schlacht als sinnlosen Wirbel, dessen Sprache die der Raben ist. Denn die Exegese des Odfeldes und der Ereignisse auf diesem Schauplatz sind Rabensprache: Sprache Raabes, Sprache des Handlungsträgers Buchius, Sprache der wirbelnden Flugfiguren der Raben. So fällt auch die textimmanente Exegese-Instanz auf das semiotische Nullniveau zurück, welches der Handlungsverlauf auf seine Weise, in der Dekonstruktion der causa finalis durch die causae efficientes, vollzogen hat.

Eine andere und nicht weniger radikale Revozierung der historistischen Kunst des Erzählens durch narrative Inversionen findet sich im Stopfkuchen, ein Text, der sich wohl insgesamt als metadiegetischer Kommentar zur historistischen Historik lesen lässt. Stopfkuchens indizienbeflissene Aufklärungsarbeit an einem vergangenen Unrecht wurde in der Forschung im Rahmen eines literarischen Schemas, der Kriminalerzählung, diskutiert. ${ }^{31}$ Der Nachweis, dass Stopfkuchen schlussendlich nur Indizien, aber keinen Beweis habe, führte folgerichtig dazu, die detektivische Arbeit als unzureichend, die literarische Kriminalform als dekonstruiert und die Ereignisrekonstruktion Stopfkuchens als Lügengeschichte zu bezeichnen. Zweifelsohne ist es wichtig, die Münchhausenanspielungen des Textes als Indiz dafür zu lesen, dass in der Tat die gesamte Erzählwelt Stopfkuchens allein sein Wille und seine Vorstellung ist. ${ }^{32}$

Aber zugleich wird man die Frage stellen müssen, ob die Spanne zwischen dem durch eine Kriminalgeschichte erforderten Beweisniveau und der in Relation dazu nur negativen Qualifizierung der Münchhausiade für diesen Text richtig ausgemessen ist. Liest man den Stopfkuchen als narrativ-reflexive Historik, dann wäre nämlich der bloße Indizienbeweis ebenso wenig eine Disqualifikation seines Geltungsanspruches, wie die Münchhausiade. Kein Historiker wird je beweisen können und keine Historik wird je den Verdacht, die Geschichtserzählung ziehe sich am eigenen narrativen Zopf aus den trüben Quellen empor, zur Gänze dementieren wollen. Als Allegorie auf die historistische Geschichts-

31 Ulf Eisele: Der Dichter und sein Detektiv. Raabes „Stopfkuchen“ und die Frage des Realismus, Tübingen 1979 (= Untersuchungen zur deutschen Literaturgeschichte, Bd. 22).

32 Johannes Graf und Gunnar Kwisinski: Heinrich Schaumann, ein Lügenbaron? Zur Erzählstruktur in Raabes „Stopfkuchen“. In: Jahrbuch der Raabe-Gesellschaft [im Folgenden JbRG] 1992, S. 194-213. 
erzählung ebenso wie als ihre permanente methodologische Verhandlung (also als Historik) liest sich der Stopfkuchen, so lautet meine These, adäquater als in der Alternative von Kriminalerzählung und Münchhausiade. Denn Stopfkuchen, selbst wenn er als Detektiv nur zu heiklen Ergebnissen kommt, agiert doch als Historiker genau so, wie Droysens Historik es bestimmt: Er sichert Quellen und bewertet sie, stellt Hypothesen über Ereignissequenzen auf, versucht diese Hypothesen zu sichern und er interpretiert, indem er eine komplette, sinnintegrierende These über den Mordfall und den möglichen Täter aufstellt. Damit nicht genug: Er erprobt sogar die soziale Relevanz seiner Gesamthypothese, indem er sie in der Dorfschenke so erzählt, dass sie sofort als Fama die Runde macht und in ihre soziale Funktion eines Gerechtigkeit stiftenden Ausgleichs eintritt. Dies alles wird durch eine permanente Reflexion des Erzählprozesses begleitet, indem Stopfkuchen die Langsamkeit seiner Erzählung, die notwendige Reihenfolge und die Schauplatzabhängigkeit der einzelnen Sequenzen gegen den Vorwurf, er lasse sich zuviel Zeit, verteidigt. „Urteil aus Ungeduld“ (Burckhardt) ${ }^{33}$ kann man ihm nicht vorwerfen, vielmehr wären seine vorbildliche Gründlichkeit, seine genaue Quellenkritik und seine permanente kommunikative Abgleichung der Zwischenergebnisse als methodologisch gesicherte Verfahrensschritte zu loben. Stopfkuchens Methodik entspricht - wie eingehender zu zeigen wäre - exakt der Historik Droysens, und sein Ergebnis geht durchaus mit dem Geltungsanspruch konform, den historische Narrative zu erheben in der Lage sind.

Aber Stopfkuchen verfolgt, indem er so streng historistisch verfährt, durchaus die Ziele der Geschichtsfälschung: Er rehabilitiert den Mörder und klagt die falsche Person an. In der präzisen Befolgung der historischen Methodik bei gleichzeitig vollständiger Verkehrung ihres Sinns, liegt, als Reflexion der Geschichtserzählung auf dritter Ebene, die poetologische Wendung gegen die Historik. Der Romantext annonciert auch dies noch. So wie im Odfeld die Kommentarinstanz der Raben das narrative Verfahren noch einmal artikulierte, wird im Stopfkuchen just der zentrale Begriff des historischen Sinns einer tiefgreifenden Dekonstruktion unterzogen, als wollte der Text den Leser mit Macht darauf stoßen, dass es hier um die literarische Reflexion des Historischen gehe. Der historische Sinn, so teilt Stopfkuchen seinem Zuhörer Eduard mit, sei ihm aufgegangen, als sich der alte Registrator Schwartner seiner annahm und ihn in die Lokalhistorie des Siebenjährigen Krieges einführte. Sinn und Zweck der historischen Studien werden von dem alten Lehrer trefflich benannt: „Ohne Geschichtskenntnis bleibt der Gescheuteste ein dummer Esel, mit ihr steckt er als überlegener Mensch eine ganze Stadt, ein ganz Gemeinwesen wissenschaftlich in die Tasche." (BA 18, S. 71) Das ist die Gebrauchsanweisung für Stopfkuchens großangelegten Betrugsversuch. Nur an der Außenseite imitiert er die investigative Recherche

\footnotetext{
33 Vgl. Anm. 18.
} 
des Detektivs; aber eigentlich ist seine Arbeit eine historische und seine Narration der ausufernde Geschichtsschmöker, der vor allem darauf ausgerichtet ist, seine Zuhörer bis zur intellektuellen Kapitulation zu langweilen, um ihnen im Moment erlahmender Aufmerksamkeit das eigentliche Erzählziel zu insinuieren. Die Langatmigkeit der Geschichtserzählung, von Burckhardt wie von Stopfkuchen als Ausweis der Wissenschaftlichkeit markiert, ist in diesem Licht eine reine Herrschaftstechnik: Man ermüde seine Zuhörer so lange, bis sie alles glauben. ${ }^{34}$ Immerhin hat diese Strategie selbst die Forschung zum Stopfkuchen über ein solides Jahrhundert hinweg lahm gelegt. Stopfkuchen lernt also die wissenschaftliche Überlegenheit über die ganze Stadt, und er lernt sie, indem er sich Überblick verschafft:

Er [Schwartner] hatte einen ziemlich großen Plan der Stadt aus dem Ende des vorigen Jahrhunderts an der Wand neben seinem Sofa hängen, und wenn er nicht draußen im Felde diese närrische verjährte Belagerung mit mir traktierte, so dozierte er sie mir von diesem Sofa aus, und ich mußte auf der Karte mit dem Finger nachfahren, meistens natürlich zwischen der Stadt und der Roten Schanze hin und her. [BA 18, S. 72]

Genau dies ist das Terrain, auf dem der Mord geschah, und Stopfkuchens ganze Indizienkonstruktion basiert darauf, dass er in der rekonstruierten Ereigniskette die kartographisch gewonnene Übersicht über die Topographie mit einer Zeitberechnung der Ereignisse so kombiniert, dass er ein Zeitintervall gewinnt, in dem er seine Version des Verbrechens stattfinden lassen kann. Historische Forschung führt zu einem konstruktiven Überblick über Raum- und Zeitverhältnisse, deren Manipulation Stopfkuchens Begehren nach Rache ebenso entgegen kommt wie der Erzählordnung des Romans. Trocken bekennt Stopfkuchen, dass es ihm in der Tat nur um diesen Ort ging und auch nicht nur um eine selbstzweckhafte Betätigung des historischen Sinns: „Daß ich mich mit ihm, immer dem historischen Sinn, einzig und allein auf die Rote Schanze zu beschränken wußte, spricht meines Erachtens zuletzt denn doch dafür, daß noch etwas in mir lag, was selbst über den historischen Sinn hinausging.“ (BA 18, S. 71)

So zeigt sich, dass der ganze Roman, dessen narrative Substanz mit Stopfkuchens Weltkonstruktion zusammenfällt, nur deshalb eine ganze Historik durchexerziert, um dem Historischen in den Rücken zu kommen. Was Stopfkuchen antreibt, ist einerseits die Rache an denen, die ihn demütigten, andererseits aber der Versuch, ein weiteres Opfer der so wenig sanftmütigen Dorfgemeinschaft durch seine historistische Münchhausiade zu rehabilitieren. Indem der alte Quakatz posthum vom Mordverdacht befreit wird, geschieht ihm Gerechtigkeit. Unmittelbar ist zu sehen, dass hier Gerechtigkeit gerade nicht aus der Geschichts-

34 Vgl. die Analyse der Erzählweise Stopfkuchens bei Maurice Haslé: Der Verdauungspastor. Magen-Sprache und peristaltische Schreibweise in Raabes „Stopfkuchen“. In: JbRG 1996, S. 92-113. 
narration folgt, sondern vielmehr nur in ihrer massiven Manipulation erzielt wird. So widerspricht Raabes literarische Historik ein weiteres Mal dem Historismus. ${ }^{35}$

Der Widerspruch entsteht, weil Raabes Poetik auf der dritten Stufe der narrativen Reflexion agiert und dort, in einer poetologischen Gegen-Wendung, die optimistischen Integrationsformen dekonstruktiv unterläuft. Raabes literarische Historik geht systematisch in die narrativen Konstituentien des geschichtlichen Sinns hinein, um sie von innen her gegen sich selbst zu wenden. Man mag dies, als pessimistische Geste, dem Einfluss Schopenhauers zuschreiben. Aber formal und reflexionstopologisch gesehen, entsteht diese poetologische Option überhaupt nur deshalb, weil das Universum der Erzählung an jedem einzelnen Punkt seiner komplexen Formation trotz formeller Stabilität immer auch inhaltlich auf die unterschiedlichste Weise realisiert werden kann. Dies ausnutzend, wird einmal mehr, nach dem alten Topos, ${ }^{36}$ die Literatur zum Kommentar der Geschichtsschreibung, weil sie berichtet, was geschehen könnte und somit dem Geschehenen in der Weise der gerechten Reflexion gegenüber stehen kann.

35 Dass er dem Historismus widerspricht, steht in wünschenswerter Deutlichkeit im Text: „Und den historischen Sinn im Menschen erklären heutzutage ja viele Gelehrte für das Vorzüglichste, was es überhaupt im Menschen gibt. Ich bin nicht dieser Ansicht." (BA 18 S. 70) Man ist versucht, an Benjamins Formel, der Historismus praktiziere die Einfühlung in die Sieger, zu denken (vgl. Walter Benjamin: Über den Begriff der Geschichte. In Ders.: Gesammelte Schriften. Hg. von Rolf Tiedemann und Hermann Schweppenhäuser, Frankfurt/Main 1980, Bd. I/2, S. 691-704, hier S. 696 [These VII]), wenn Stopfkuchens bewusste Manipulation der Ereignisse mithilfe der methodengenauen historischen Recherche das Ziel hat, gegen die Faktenlage eine individuelle Gerechtigkeit zu ermöglichen.

36 Aristoteles: Poetik. Hg. von Manfred Fuhrmann, Stuttgart 1982, S. 29 (Kapitel 9). 